\title{
POLYNOMIAL INTEGRALS OF HAMILTONIAN SYSTEMS WITH EXPONENTIAL INTERACTION
}

UDC $517.9+531.01$

\author{
V. V. KOZLOV AND D. V. TRESHCHEV
}

\begin{abstract}
The problem on the complete integrability of Hamiltonian systems with exponential interaction is considered. These systems include, in particular, Toda chains and their generalizations. Conditions for the existence of a complete set of independent polynomial integrals are found. A complete classification of integrable systems is given by means of Dynkin diagrams. Certain new integrable chains are indicated.

Bibliography: 20 titles.
\end{abstract}

\section{Introduction}

Let $W$ and $W^{*}$ be a dual $n$-dimensional linear spaces over the reals. We shall denote their elements by $x$ and $y$ respectively. Let $(y, x)$ be the value of the covector $y$ on the vector $x$. Let us consider the function $V: W \rightarrow \mathbf{R}$ defined by

$$
V(x)=\sum_{k=1}^{m} v_{k} \exp \left(a_{k}, x\right),
$$

where the $v_{k}$ are nonzero real numbers and $a_{1}, \ldots, a_{m}$ are nonzero vectors from $W^{*}$. The function $V$ plays the role of the potential energy of the potential of an exponential interaction. We denote by $\mathfrak{M}$ the collection of vectors $a_{1}, \ldots, a_{m}$, the "spectrum" of the sum of exponents (1). Let $\langle$,$\rangle be an inner product in$ the space $W^{*}$. The metric $\langle$,$\rangle allows one to identify the dual spaces W$ and $W^{*}$. More precisely, there exists a linear isomorphism $A: W^{*} \rightarrow W$ such that $(y, x)=\left\langle y, A^{-1} x\right\rangle$ for every $x \in W$ and $y \in W^{*}$. Knowing the metric $\langle$,$\rangle and$ the potential $V$, we now can write down the equations of motions of a system with the exponential interaction $(W,\langle\rangle, V$,$) :$

$$
\dot{x}=A y, \quad \dot{y}=-\sum_{k}\left[v_{k} \exp \left(a_{k}, x\right)\right] a_{k} .
$$

Let $e_{1}, \ldots, e_{n}$ and $e_{1}^{*}, \ldots, e_{n}^{*}$ be dual bases in $W$ and $W^{*}$. We set $s=\sum x_{i} e_{i}$ and $y=\sum y_{i} e_{i}^{*}$. In the coordinates $x_{1}, \ldots, x_{n}$ and $y_{1}, \ldots, y_{n}$ on the phase space $W \times W^{*}$ the equations (2) may be written as Hamilton's canonical equations

$$
\dot{x}_{i}=\partial H / \partial y_{i}, \quad \dot{y}_{i}=-\partial H / \partial x_{i}, \quad i=1, \ldots, n,
$$

1980 Mathematics Subject Classification (1985 Revision). Primary 58F07, 58F05; Secondary 70H05, $34 \mathrm{~A} 25$. 
with Hamiltonian $H=T+V$, where $T=\langle y, y\rangle / 2$ is the kinetic energy of the system.

Let $D: W \rightarrow W$ be a nondegenerate linear operator, and let $D^{*}: W^{*} \rightarrow W^{*}$ be the adjoint operator. The map $W \times W^{*} \rightarrow W \times W^{*}$ given by the formulas $x^{\prime}=D x$ and $y^{\prime}=\left(D^{*}\right)^{-1} y$ is canonical. In particular, in the new variables $x_{1}^{\prime}, \ldots, x_{n}^{\prime}$, $y_{1}^{\prime}, \ldots, y_{n}^{\prime}$ the Hamiltonian equations (3) will have again the canonical form with the same Hamiltonian. By a suitable choice of $D$ we may reduce the kinetic energy to a sum of squares:

$$
T=\frac{1}{2}\left(y_{1}^{2}+\cdots+y_{n}^{2}\right) .
$$

Hamiltonian systems of the form (2) are frequently encountered in applications. For instance, the dynamics of a finite periodic Toda chain [1] is described by a system (3) with the Hamiltonian function

$$
H=\frac{1}{2} \sum_{s=1}^{n} y_{s}^{2}+\sum_{s=1}^{n} \exp \left(x_{s}-x_{s+1}\right), \quad x_{n+1}=x_{1} .
$$

Equations (2) are also encountered in the study of certain homogeneous cosmological models in general relativity theory [2].

Many papers are devoted to the search of cases of integrability of Hamiltonian systems (2). Hénon [3], Flaschka [4], and Manakov [5] established the complete integrability of the Toda chain: the Hamilton equations with Hamiltonian (4) have $n$ independent first integrals, polynomial in the impulses, that are pairwise in involution. This result was generalized in [6]-[8] to the case when the spectrum of $\mathfrak{M}$ is a system of simple roots of a simple Lie algebra. From this point of view, to the Hamiltonian (4) there corresponds an algebra of type $A_{n}$. Sklyanin [9] indicated another integrable generalization of the Toda chain:

$$
\begin{aligned}
H= & \sum_{s=1}^{n} \frac{y_{s}^{2}}{2}+\sum_{s=1}^{n-1} \exp \left(x_{s+1}-x_{s}\right)+\alpha_{1} \exp x_{1}+\frac{\beta_{1}}{2} \exp \left(2 x_{1}\right) \\
& +\alpha_{n} \exp \left(-x_{n}\right)+\frac{\beta_{n}}{2} \exp \left(-2 x_{n}\right),
\end{aligned}
$$

where $\alpha_{1}, \beta_{1}, \alpha_{n}$, and $\beta_{n}$ are arbitrary real constants. The method of [3]-[9] is based on the representation of Hamilton's equations (2) as an $L-A$ Lax pair. The entries of the matrices $L$ and $A$ are linear functions of the impulses $y_{1}, \ldots, y_{n}$ whose coefficients are finite sums of real exponents:

$$
\sum f_{\lambda} \exp \left(c_{\lambda}, x\right), \quad f_{\lambda} \in \mathbf{R}, c_{\lambda} \in \mathbf{R}^{n} .
$$

Consequently, the traces of the powers of the matrix $L$-the integrals of Hamilton's equations- are polynomials in the impulses with coefficients of the form (6).

In the general case little is known about the integrability of systems (2). In [10] the case was considered when $\mathfrak{M}$ consists of $n+1$ vectors $a_{1}, \ldots, a_{n+1}$, and any $n$ of them are assumed to be independent. It is proved that under these assumptions a criterion for the algebraic integrability of $(2)$ is that

$$
\frac{2\left\langle a_{i}, a_{j}\right\rangle}{\left\langle a_{i}, a_{i}\right\rangle} \in-\mathbf{Z}^{+}, \quad \mathbf{Z}^{+}=\{0,1,2, \ldots\}
$$

for every $i \neq j$. The algebraic integrability means, in particular, that the variables $y_{s}$ and $\exp x_{s}(1 \leq s \leq n)$ are meromorphic on the plane of complex time for 
almost every initial data. The search for necessary algebraic integrability conditions is based on a classical method of Kovalevskaya that she used in solid dynamics [11]. We observe that far from every completely integrable system of the form (2) is algebraically integrable in the sense of the definition [10]. A simple example with one degree of freedom is

$$
H=\left[y^{2}+\exp (-2 x) f_{m}(\exp x)\right] / 2,
$$

where $f_{m}(\cdot)$ is a polynomial of degree $m$ with simple roots. This system is not algebraically integrable for $m \geq 5$. Indeed, the functions

$$
\int\left(2 h q^{2}-f_{m}(q)\right)^{-1 / 2} d q=t, \quad q=\exp x, \quad y=\dot{q} / q
$$

are solutions with a total energy reserve of $h$. Clearly, for $m \geq 5$ for almost every $h$ the function $t \mapsto q(t)$ is multivalued on the complex plane.

We shall study the integrability of (2) in the real domain.

In [6] Bogoyavlensky has shown the complicated behavior of the trajectories of (2) with an infinite Coxeter group generated by reflections with respect to the vectors $a \in \mathfrak{M}$. It would be interesting to relate the finiteness of the Coxeter groups with the existence of an additional integral independent of the energy $H$.

\section{§1. Structure of integrable systems}

A Hamiltonian system of equations (2) is called Birkhoff integrable if it has $n$ integrals, polynomial with respect to the impulses, with coefficients of the form (6) whose leading homogeneous forms are almost everywhere independent (as functions in $W \times W^{*}$ ).

In the case of two degrees of freedom we replace the independence condition of the leading forms by the weaker condition of independence of an additional polynomial integral of the energy integral (as analytic functions in the phase space $W \times W^{*}$ ).

Birkhoff studied the conditions for the existence of linear and quadratic integrals in the impulses of general natural Hamiltonian systems with two degrees of freedom [12]. The existence of these integrals is connected with the existence of "hidden" cyclic coordinates and separated canonical variables. The Hamiltonian systems in [3]-[9] are Birkhoff integrable, as was observed above.

We shall say that a vector in $\mathfrak{M}$ is maximal if it has the greatest possible length among all the vectors of $\mathfrak{M}$ having the same direction.

Our main result is the following

THeorem 1. Assume that the Hamiltonian system (2) is Birkhoffintegrable. Let $a_{i}$ be a maximal vector in $\mathfrak{M}$ and assume that the vector $a_{j} \in \mathfrak{M}$ is linearly independent of $a_{i}$. Then

$$
\frac{2\left\langle a_{i}, a_{j}\right\rangle}{\left\langle a_{i}, a_{i}\right\rangle} \in-\mathbf{Z}^{+}
$$

COROLLARY 1. If system (2) is Birkhoff integrable, then any two linearly independent maximal vectors $a_{i}, a_{j} \in \mathfrak{M}$ satisfy condition (7).

It is convenient to compare this assertion with the result of [10], which considered the case when $\mathfrak{M}$ consists of $n+1$ vectors $a_{1}, \ldots, a_{n+1}$ such that any subsystem of $n$ vectors is linearly independent. In [10] it was shown that a criterion of algebraic integrability of (2) is precisely the fulfillment of (7). Corollary 1 states that in this case a criterion of Birkhoff integrability is condition (7) as well. This situation is 
analogous to the state of affairs in the classical problem on the rotation of a heavy rigid body with a fixed point: the equations of motion are algebraically integrable if and only if they have a complete set of independent polynomial integrals (see, for instance, [11]).

COROLLARY 2. If the system is Birkhoff integrable, then the following assertions are true.

1) The angle between any two vectors of $\mathfrak{M}$ has one of the following values: 0 , $\pi / 2,2 \pi / 3,3 \pi / 4,5 \pi / 6$, or $\pi$.

2) Let $a, a^{\prime} \in \mathfrak{M}$ and assume that the vector $a$ is maximal. If the angle between $a$ and $a^{\prime}$ is equal to $2 \pi / 3$ then $|a|=\left|a^{\prime}\right|$; if it is $3 \pi / 4$ then either $|a|=\sqrt{2}\left|a^{\prime}\right|$ or $|a|=\left|a^{\prime}\right| / \sqrt{2}$; and if it is $5 \pi / 6$ then either $|a|=\sqrt{3}\left|a^{\prime}\right|$, or $|a|=2\left|a^{\prime}\right| \sqrt{3}$, or $|a|=\left|a^{\prime}\right| / \sqrt{3}$.

The deduction of 1) from condition (7) is well known in the theory of root systems (see, for instance, [13] and [14]). Conclusion 2) is proved in $\S 2$.

We give below the classification of Birkhoff integrable Hamiltonian systems.

Assume that $W^{*}$ is the direct sum of the orthogonal subspaces $W_{1}^{*}, \ldots, W_{m}^{*}$ and the spectrum of $\mathfrak{M}$ lies in their union $\cup W_{l}^{*}$. Denote by $W_{1}, \ldots, W_{m}$ the images of $W_{1}^{*}, \ldots, W_{m}^{*}$ under the map $A: W^{*} \rightarrow W$. It is easy to see that system (2) splits into $m$ closed subsystems with phase spaces $W_{i} \times W_{i}^{*} \subset W \times W^{*}$. Let $H_{i}$ be the restriction of the Hamiltonian function $H$ to $W_{i} \times W_{i}^{*}$. Then, clearly, $H=\sum H_{i}$. If the basis vectors $e_{1}, \ldots, e_{n}$ belong to the union $W_{1} \cup \cdots \cup W_{m}$, then in the corresponding canonical variables $x_{1}, \ldots, x_{n}, y_{1}, \ldots, y_{n}$ equations (3) split into $m$ closed Hamiltonian systems with Hamiltonian functions $H_{i}$ (i.e., we have a partial separation of variables). We shall say that in this situation the original Hamiltonian system is a direct sum of its subsystems. If such splitting is impossible (clearly, under the assumption that each subspace $W_{i}$ is nontrivial), then we shall say that the Hamiltonian system is irreducible. The following assertion is clear.

Proposition 1. Each Hamiltonian system (2) is the direct sum of its irreducible Hamiltonian subsystems.

Let $\alpha$ and $\beta$ be vectors from $\mathfrak{M}$ and let $\varphi$ be the angle between them. If the Hamiltonian system is Birkhoff integrable then, as stated in Corollary 2 to Theorem $1,4 \cos ^{2} \varphi$ may be one of the following integers: $0,1,2,3$, or 4 . This fact suggests that we should introduce the Coxeter graph of the Birkhoff integrable Hamiltonian system. This is a graph whose vertices are the vectors of $\mathfrak{M}$, and two vertices $\alpha$ and $\beta$ are joined by $4 \cos ^{2} \varphi$ edges. Clearly, if the integrable system is irreducible then its Coxeter graph is connected and nonempty.

If $\operatorname{dim} W_{i}^{*}=1$, then $\mathfrak{M} \cap W_{i}^{*}$ may be any finite set of vectors. This remark follows from the complete integrability of Hamiltonian systems with one degree of freedom. Leaving aside these trivial cases, we assume below that $\operatorname{dim} W_{i}^{*}>1$.

Thus, let us consider the structure of an integrable irreducible Hamiltonian system with $n>1$ degrees of freedom.

Proposition 2. Any two linearly dependent vectors of $\mathfrak{M}$ have the same direction.

Proof. Let us set $\Pi_{\alpha}=\left\{b \in W^{*}:\langle\alpha, b\rangle \leq 0\right\}$. Let $\alpha$ and $\beta$ be linearly dependent vectors of $\mathfrak{M}$. If $\gamma \in \mathfrak{M}$ and $\gamma \neq k \alpha, k \in \mathbf{R}$, then by Theorem 1 we have $\gamma \in \Pi_{\alpha}$ and $\gamma \in \Pi_{\beta}$. If $\alpha$ and $\beta$ have opposite directions, then the intersection $\Pi_{\alpha} \cap \Pi_{\beta}$ is a hyperplane in $W^{*}$ orthogonal to both $\alpha$ and $\beta$. Therefore, in this 
case $\gamma$ is orthogonal to the vectors $\alpha$ and $\beta$. But this contradicts the irreducibility assumption. The proposition is proved.

We say that an integrable system with spectrum $\mathfrak{M}$ is complete if there is no nonzero vector $a \in W^{*}$ such that the set $\mathfrak{M} \cup\{a\}$ satisfies the assumptions of Theorem 1. The spectrum of each Birkhoff integrable Hamiltonian system is obtained from a complete spectrum by dropping part of the elements. Of course, in this reduction of $\mathfrak{M}$ we must preserve the connectedness of the Coxeter graph, and the number of its vertices cannot be less than $\operatorname{dim} W^{*}=n$.

As is easily understood, the Coxeter graph determines only the angles between pairs of vectors of $\mathfrak{M}$. In order to be able to reconstruct the ratios of the lengths of the vectors we assign to every vertex of the Coxeter graph a coefficient proportional to the square of the length $\langle a, a\rangle$ of the corresponding vector $a \in \mathfrak{M}$. This extended Coxeter graph will be called a Dynkin scheme (as is customary in the theory of root systems). We agree to identify Dynkin schemes differing only by a positive proportionality coefficient. This convention can be given a clear dynamical meaning.

Let us consider two dynamical systems such that the vectors of their spectra differ by a positive factor $k>0$. It is not hard to check that the change $x \mapsto x k, t \mapsto k t$ carries the equations of motion (2) of one system into those of the other. Using Corollary 2 to Theorem 1 and Proposition 2, one can prove that the Dynkin scheme uniquely (up to an isomorphism) determines the spectrum of an integrable irreducible Hamiltonian system (cf. [13] and [14]).

THeOREM 2. The Dynkin scheme of a complete irreducible Birkhoff integrable Hamiltonian system is isomorphic to one of the following schemes:

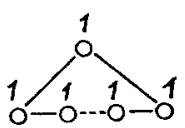

a

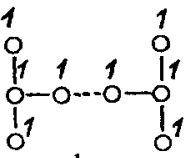

b

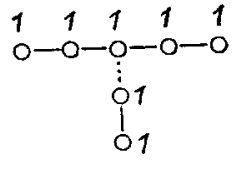

c

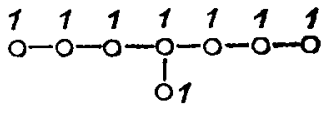

$\mathrm{d}$

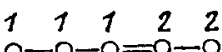

$\mathrm{f}$
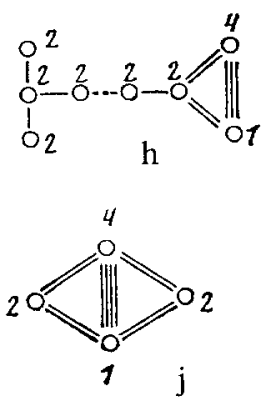

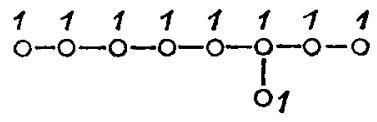

e

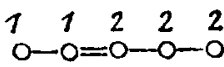

$\mathrm{g}$
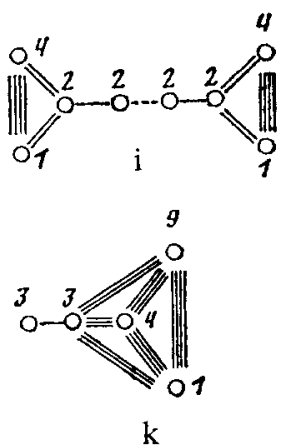
COROLlary. The spectrum of an irreducible Birkhoff integrable Hamiltonian system with $n \geq 2$ degrees of freedom contains at most $n+3$ distinct vectors.

The estimate card $\mathfrak{M} \leq n+3$ cannot be improved. This is shown by the example of a system with Hamiltonian function (5).

In order to prove Theorem 2 we shall need the following fact.

LEMma 1. Assume that the vectors $a_{\lambda} \in \mathfrak{M}$ are pairwise linearly independent and $\sum x_{\lambda} a_{\lambda}=0$ for some real $x_{\lambda} \neq 0$. If $a$ is a vector of $\mathfrak{M}$ linearly independent from each vector $a_{\lambda}$, then $\left\langle a, a_{\lambda}\right\rangle=0$.

Proof. The equality $\sum x_{\lambda} a_{\lambda}=0, x_{\lambda} \neq 0$, may be rewritten as

$$
\sum y_{\mu} a_{\mu}=\sum z_{\nu} a_{\nu}, \quad y_{\mu}, z_{\nu}>0 .
$$

Let us set $b=\sum y_{\mu} a_{\mu}$ and compute

$$
\langle b, b\rangle=\sum y_{\mu} z_{\nu}\left\langle a_{\mu}, a_{\nu}\right\rangle .
$$

Since the vectors $a_{\lambda}$ are pairwise linearly independent, by Theorem 1 we have $\langle b, b\rangle$ $\leq 0$. Hence $b=0$. Since

$$
0=\langle a, b\rangle=\sum y_{\mu}\left\langle a, a_{\mu}\right\rangle, \quad y_{\mu}>0,
$$

and $\left\langle a, a_{\mu}\right\rangle \leq 0$ (Theorem 1), we clearly have $\left\langle a, a_{\mu}\right\rangle=0$. Analogously $\left\langle a, a_{\nu}\right\rangle=$ 0 , as required.

Proposition 3. The set $\mathfrak{M}$ contains no more than $n+1$ pairwise linearly independent vectors, and any $n$ of them are linearly independent.

Proof. Let $\mathfrak{M}$ have $n+1$ pairwise independent vectors $a_{1}, \ldots, a_{n+1}$. Since $\operatorname{dim} W^{*}=n$, we have

$$
\sum_{i=1}^{n+1} x_{i} a_{i}=0, \quad \sum x_{i}^{2} \neq 0 .
$$

Let $\mathfrak{M}^{\prime}$ denote the set of vectors of $\mathfrak{M}$ that are dependent with those $a_{1}, \ldots, a_{n+1}$ for which $x_{i} \neq 0$. By Lemma 1 , the sets $\mathfrak{M}^{\prime}$ and $\mathfrak{M} \backslash \mathfrak{M}^{\prime}$ are orthogonal. Since by assumption the system is irreducible, it follows that $\mathfrak{M}=\mathfrak{M}^{\prime}$ and all the $x_{i}$ are nonzero. In particular, any $n$ vectors from the set $a_{1}, \ldots, a_{n+1}$ are linearly independent. The proposition is proved.

There is a complete classification of systems of $n+1$ vectors in $n$-dimensional Euclidean space satisfying (7) and such that each proper subsystem is linearly independent (see [15], and also [10]). Such systems are systems of simple roots of graded Kac-Moody algebras. The complete Dynkin diagrams a)-k) enumerated in Theorem 2 were obtained from the well-known root systems of Kac-Moody algebras, taking into account the fact that the spectrum of an integrable system may contain vectors with the same direction. We omit the simple arguments. Assume now that $\mathfrak{M}$ contains $n$ linearly independent maximal vectors satisfying condition (7). It turns out that such a system is not complete in the sense of our definition: to these $n$ vectors we can add one vector so that condition (7) will be preserved and any subsystem of $n$ vectors will be linearly independent. This follows, for instance, from the fact that a Dynkin diagram of a system of simple roots is obtained from a diagram of root system of certain Kac-Moody algebra by dropping one vertex [13]-[15]. 
Now let us consider the question on the Birkhoff integrability of the Hamiltonian systems enumerated in Theorem 2 . In the cases a) $-\mathrm{g}$ ) the complete integrability was established in [6] and [16]. The Hamiltonian of a system with a Dynkin scheme i) can always be reduced to the form (5). This system has been integrated in [9].

The graph j) corresponds to a Hamiltonian system with two degrees of freedom with Hamiltonian functions

$$
\begin{gathered}
H=\frac{1}{2}\left(y_{1}^{2}+y_{2}^{2}\right)+v_{1} e_{1}+v_{2} e_{2}+v_{3} e_{3}+v_{4} e_{4}, \\
e_{1}=\exp x_{1}, \quad e_{2}=\exp x_{2}, \quad e_{3}=\exp \left(-x_{1}-x_{2}\right), \quad e_{4}=\exp \left(-\frac{x_{1}+x_{2}}{2}\right) .
\end{gathered}
$$

For all the values of the coefficients $v_{1}, \ldots, v_{4}$ it has an additional integral of fourth degree with respect to the impulses:

$$
\begin{aligned}
F= & y_{1}^{2} y_{2}^{2}+2 v_{2} y_{1}^{2} e_{2}+2 v_{3} y_{1} y_{2} e_{3}+2 v_{4} y_{1} y_{2} e_{4}+2 v_{1} y_{2}^{2} e_{1} \\
& +2 v_{2} v_{3} e_{2} e_{3}+2 v_{1} v_{3} e_{1} e_{3}+4 v_{1} v_{2} e_{1} e_{2}+v_{3}^{2} e_{3}^{2}+2 v_{3} v_{4} e_{3} e_{4}+v_{4}^{2} e_{4}^{3} .
\end{aligned}
$$

This is a new integrable chain. If $v_{3}=0$ or $v_{4}=0$, we obtain the integrable chains of [6] and [16]. Clearly, the leading homogeneous forms of the functions $H$ and $F$ are independent.

A Hamiltonian function with Dynkin scheme h) has the following form in suitable canonical variables:

$$
\begin{aligned}
H= & \frac{1}{2} \sum_{s=1}^{n} y_{s}^{2}+\sum_{s=1}^{n-1} \exp \left(x_{s}-x_{s+1}\right)+\exp \left(-x_{1}-x_{2}\right) \\
& +\alpha_{n} \exp x_{n}+\beta_{n} \exp \left(2 x_{n}\right) ; \quad \alpha_{n}, \beta_{n} \in \mathbf{R}, n \geq 4 .
\end{aligned}
$$

If either $\alpha_{n}=0$ or $\beta_{n}=0$, then the complete integrability of this system has been established in [6] and [16]: when $\beta_{n}=0$ this is a system of type $B_{n}$ (by the classification in [6]), and when $\alpha_{n}=0$ we obtain a system of type $b_{1}^{(1)}$ (by the classification in [10]). The question of the integrability of the Hamiltonian equations with Hamiltonian (8) in the general case, when $\alpha_{n} \neq 0$ and $\beta_{n} \neq 0$, remained unclear. The answer to this question is apparently positive.

The question on the integrability of a Hamiltonian system with a graph $\mathrm{k}$ ) turned out to be more complicated. In [6] and [16] Hamiltonian systems were integrated with two degrees of freedom whose oriented Coxeter graph has the following form:
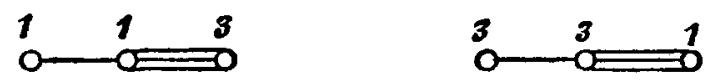

They are obtained from the graph $\mathrm{k}$ ) by eliminating two vertices. We have studied the problem on the Birkhoff integrability of a system with Hamiltonian

$$
\begin{gathered}
H=\frac{1}{2}\left(y_{1}^{2}+y_{2}^{2}\right)+v_{1} \exp x_{1}+v_{2} \exp \left(-\frac{1}{2} x_{1}-\sqrt{3} x_{2} / 2\right) \\
+v_{3} \exp \left(\sqrt{3} x_{2} / 3\right)+v_{4} \exp \left(\sqrt{3} x_{2}\right) .
\end{gathered}
$$

Its Coxeter graph is obtained from that of $k$ ) by dropping one vertex. This case differs from the general one in that all the linearly independent vectors from $\mathfrak{M}$ satisfy (7). It turned out that if all the coefficients $v_{i}$ are nonzero then the Hamilton equations with Hamiltonian (9) do not have an additional integral whose power does 
not exceed 6. The number 6 is not accidental: this is the rank of the Coxeter group generated by reflections with respect to vectors in the spectrum of $\mathfrak{M}$. We observe that in the remaining integrable systems with two degrees of freedom the degree of the additional polynomial integral is precisely equal to the rank of the corresponding Coxeter group. Apparently a system with Hamiltonian (9) (and all the more a system with graph $\mathrm{k}$ )) is not Birkhoff integrable.

\section{§2. Necessary integrability conditions}

Let $e_{1}, \ldots, e_{n}$ and $e_{1}^{*}, \ldots, e_{n}^{*}$ be dual bases in $W$ and $W^{*}$. We introduce an order relation in $W^{*}$, which we shall denote by $\prec$. Let $\sigma=\sum \sigma_{i} e_{i}^{*}$ and $\delta=\sum \delta_{i} e_{i}^{*}$. We say that $\sigma \preccurlyeq \delta$ if for the least index $k$ such that $\sigma_{k} \neq \delta_{k}$ we have $\sigma_{k}<\delta_{k}$. Clearly, $\prec$ is the usual lexicorgraphic order relation in $W^{*}$ in the basis $e_{1}^{*}, \ldots, e_{n}^{*}$. This relation induces in a natural way an order relation in $W$. We say that $\sigma \preceq \delta$ if either $\sigma \prec \delta$ or $\sigma=\delta$.

The proof of Theorem 1 uses the following assertion, which has independent interest.

THEOREM 3. Let $\alpha$ be a maximal element in $\mathfrak{M}$ with respect to the order relation $\prec$. Assume that there is a vector $\beta \in \mathfrak{M}$ satisfying the following conditions:

(1) $\beta>0$ and $\beta$ is independent of $\alpha$.

(2) The equality $s \alpha+\beta=\tau_{1}+\cdots+\tau_{s+1}$, where $\tau_{i} \in \mathfrak{M}$, implies $\tau_{k}=\beta$ and $\tau_{j}=\alpha \quad(j \neq k)$.

(3) $m\langle\alpha, \alpha\rangle+2\langle\alpha, \beta\rangle \neq 0$ for all integers $m \geq 0$.

Then the Hamiltonian system (2) is not Birkhoff integrable.

We make two remarks.

1) For condition (2) to hold it suffices to require that $\beta$ be a maximal vector of $\mathfrak{M}$ linearly independent of $\alpha$.

2) Theorem 3 is also valid when the metric $\langle$,$\rangle is pseudo-Euclidean.$

Theorem 3 will be proved in $\S 3$. Now we will deduce Theorem 1 from it.

On the vector $W^{*}$ we may introduce a natural affine structure; therefore, $\mathfrak{M}$ may be regarded as a finite collection of points of an affine space. We denote by $\mathscr{E}(\mathfrak{M})$ the convex hull of $\mathfrak{M}$. Clearly, $\mathscr{E}(\mathfrak{M})$ is a convex polyhedron in the $n$-dimensional affine space. The necessary notions from the theory of convex figures may be found, for instance, in [17].

LEMMA 2. Let $\alpha$ and $\beta$ be two adjacent vertices of $\mathscr{E}(\mathfrak{M})$ (adjacent by an edge), linearly independent as vectors of $W^{*}$. If the Hamiltonian system is integrable, then for some integer $m \geq 0$ we have $m\langle\alpha, \alpha\rangle+2\langle\alpha, \beta\rangle=0$.

Proof. Let $\gamma$ be a segment joining $\alpha$ and $\beta$. Clearly, $\gamma$ is an edge of the convex polyhedron $\mathscr{E}(\mathfrak{M})$. Since $\alpha$ and $\beta$, as vectors of $W^{*}$, are linearly independent and $\mathscr{E}(\mathfrak{M})$ is a convex polyhedron, then there exists a hyperplane $\Gamma$ not containing $0 \in W^{*}$ such that $\Gamma \cap \mathscr{E}(\mathfrak{M})$ coincides with $\Gamma$. There are two possibilities: either $\Gamma$ separates the polyhedron $\mathscr{E}(\mathfrak{M})$ and the point $0 \in W^{*}$, or it does not separate them. In the first case we choose the following basis in $W^{*}: e_{1}^{*}=\alpha, \varepsilon_{2}^{*}$ is the vector starting at $\beta$ and ending at $\alpha$, and $e_{3}^{*}, \ldots, e_{n}^{*}$ are vectors in $\Gamma$ linearly independent of $e_{2}^{*}$. In the second case we choose the vectors $\left(-e_{1}^{*}\right), e_{2}^{*}, \ldots, e_{n}^{*}$ as the basis.

Clearly, in this basis the vector $\alpha$ is the maximal element of the set $\mathfrak{M}$. Let $\alpha^{\prime}$ be the greatest element in $\mathfrak{M} \backslash\{\alpha\}$. Clearly, $\alpha^{\prime}$, as a point of the affine space, is 
the nearest point to $\alpha$ in the set $(\mathfrak{M} \backslash\{\alpha\}) \cap \Gamma$. We claim that the angle between $\alpha$ and $\alpha^{\prime}$ is not less than $\pi / 2$. Indeed, otherwise both $\alpha$ and $\alpha^{\prime}$ satisfy conditions (1) $-(3)$ of Theorem 3, and so the Hamiltonian system under consideration is not integrable. Let $\beta^{\prime}$ be the nearest point to $\beta$ in $(\mathfrak{M} \backslash\{\beta\}) \cap \gamma$. We prove analogously that the angle between $\beta$ and $\beta^{\prime}$ is not less than $\pi / 2$. Since the vectors $\alpha$ and $\beta$ are independent by assumption, the angle between them is strictly less than $\pi$. Consequently, the point $\alpha^{\prime}$ actually coincides with the vertex $\beta$, and $\beta^{\prime}$ coincides with $\alpha$. Hence it follows that the vectors $\alpha$ and $\beta$ satisfy conditions (1) and (2) of Theorem 3. If (3) also holds, the system will be nonintegrable, against the assumption. The lemma is proved.

Lemma 3. Assume that the original Hamiltonian system is not Birkhoff integrable. Then the following assertions are true:

(1) All the points of $\mathfrak{M}$ lie on rays starting at $0 \in W^{*}$ and passing through the vertices of the convex polyhedron $\mathscr{E}(\mathfrak{M})$,

(2) The angles between these rays are not less than $\pi / 2$.

Proof. Let $\delta$ be a point of $\mathfrak{M}$. Then there is a vertex $\sigma$ of the polyhedron $\mathscr{E}(\mathfrak{M})$ such that the angle between $\sigma$ and $\delta$ is acute. For otherwise all the vertices lie in the half-space $\Pi_{\delta}=\left\{y \in W^{*}:\langle y, \delta\rangle \leq 0\right\}$. Hence, $\mathscr{E}(\mathfrak{M}) \subset \Pi_{\delta}$, and therefore $\delta \notin \mathscr{E}(\mathfrak{M})$. We reach a contradiction. We shall assume that $\delta$ does not lie on the ray given by the vector $\sigma$. Then the angle between $\sigma$ and $\delta$ is nonzero. We may also assume that $\sigma$ has maximal length among all the vectors of $\mathfrak{M}$ with the same direction as $\sigma$.

Let $\Gamma$ be the hyperplane passing through $\sigma$ (as a point of affine space) and orthogonal to the vector $\sigma$. Let us denote by $\Pi$ the closed half-space with boundary $\Gamma$ which does not contain 0 . We show that $\mathscr{E}(\mathfrak{M})$ has only the point $\sigma$ in common with $\Pi$. Indeed, otherwise there is another point $\tau \neq \sigma$. But then there is a point $\mu$, a vertex of $\mathscr{E}(\mathfrak{M})$, that is connected to $\sigma$ by an edge and lies in $\Pi$. Let us prove this assertion by contradiction. Clearly, $\mathscr{E}(\mathfrak{M})=M_{1} \cup M_{2}$, where $M_{1}$ is the convex hull of the vertices of $\mathscr{E}(\mathfrak{M})$ except $\sigma$, and $M_{2}$ is the convex hull of the edges issuing from $\sigma$.

Let us consider the segment $\gamma$ joining $\sigma$ and $\tau$. Assume there is no such point $\mu$. Then $\gamma \cap M_{2}=\Pi \cap M_{2}=\sigma$, the set $\gamma \cap M_{1}$ is closed and does not contain $\sigma$. But this contradicts the relation $\gamma \subset\left(M_{1} \cup M_{2}\right)$. Thus, there is indeed such a point $\mu$. The angle between $\sigma$ and $\mu$ is acute and nonzero. Consequently, by Lemma 2, the system is nonintegrable.

Thus, $\mathscr{E}(\mathfrak{M}) \cap \Pi=\{\sigma\}$. Let us consider the following basis $\left\{e_{i}^{*}\right\}$ in $W^{*}: e_{1}^{*}=$ $\sigma$, and $e_{2}^{*}, \ldots, e_{n}^{*}$ are independent vectors in $\Pi$. Let $\prec$ be the corresponding lexicographic order relation. Clearly, $\sigma$ is a maximal element in $\mathfrak{M}$ and $\delta \succ 0$. Let $\delta^{\prime}$ be the maximal element of $\mathfrak{M}$ that is linearly independent from $\sigma$. Since $\delta^{\prime} \succeq \delta$, we have $\left\langle\sigma, \delta^{\prime}\right\rangle>0$ and therefore the angle between $\sigma$ and $\delta^{\prime}$ is also acute. By Remark 1 after Theorem 3 the system is nonintegrable. The lemma is proved.

Now it is not hard to prove Theorem 1. By Lemma 3, for any two linearly independent vectors $\sigma$ and $\tau$ of $\mathfrak{M}$ we have $\langle\sigma, \tau\rangle \leq 0$. Let $\alpha$ and $\beta$ be linearly independent vectors of $\mathfrak{M}$, and assume that $\alpha$ is maximal. Let us choose the following basis $\left\{e_{i}^{*}\right\}$ in $W^{*}: e_{1}^{*}=\alpha, e_{2}^{*}=\beta$, and the vectors $e_{3}^{*}, \ldots, e_{n}^{*}$ are orthogonal to $\alpha$ and $\beta$. Let $\tau=\sum \tau_{i} e_{i}^{*} \in \mathfrak{M}$. Let us prove that $\tau_{1} \leq 0$ if $\tau$ and $\alpha$ are linearly 
independent. Indeed,

$$
\begin{aligned}
& \langle\tau, \alpha\rangle=\tau_{1}\left\langle e_{1}^{*}, e_{1}^{*}\right\rangle+\tau_{2}\left\langle e_{2}^{*}, e_{1}^{*}\right\rangle \leq 0, \\
& \langle\tau, \beta\rangle=\tau_{1}\left\langle e_{1}^{*}, e_{2}^{*}\right\rangle+\tau_{2}\left\langle e_{2}^{*}, e_{2}^{*}\right\rangle \leq 0 .
\end{aligned}
$$

If $\tau_{1}>0$, then the first inequality implies $\tau_{2}>0$. Therefore,

$$
\tau_{1}\left\langle\tau, e_{1}^{*}\right\rangle+\tau_{2}\left\langle\tau, e_{2}^{*}\right\rangle \leq 0 \text {. }
$$

On the other hand, the last inequality may be written as

$$
\tau_{1}^{2}\left\langle e_{1}^{*}, e_{1}^{*}\right\rangle+2 \tau_{1} \tau_{2}\left\langle e_{1}^{*}, e_{2}^{*}\right\rangle+\tau_{2}^{2}\left\langle e_{2}^{*}, e_{2}^{*}\right\rangle \leq 0 .
$$

However, this contradicts the positive definiteness of the Gram matrix $\left\|\left\langle e_{i}^{*}, e_{j}^{*}\right\rangle\right\|$, $i, j=1,2$.

Thus, the order relation $\prec$ corresponding to the basis $\left\{\boldsymbol{e}_{i}^{*}\right\}$ is such that $\alpha$ is the greatest element of $\mathfrak{M}$ and $\beta \succ 0$. Moreover, if $\tau \geqslant \beta$ then $\tau$ has the same direction as either $\alpha$ or $\beta$. These properties of the vectors $\alpha$ and $\beta$ imply conditions (1) and (2) of Theorem 3. Thus, by Theorem 3, if the Hamiltonian system is integrable then for some integer $m \geq 0$ we have

$$
m\langle\alpha, \alpha\rangle+2\langle\alpha, \beta\rangle=0 .
$$

Now let $\beta$ be the vector in $\mathfrak{M}$ of maximal length having the same direction as $\beta$. For some integer $k \geq 0$ we obtain the analogous relation

$$
k\langle\beta, \beta\rangle+2\langle\beta, \alpha\rangle=0 .
$$

from (10) and (11) we conclude that the angle $\varphi$ between $\alpha$ and $\beta$ has one of the following values: $2 \pi / 3,3 \pi / 4$, or $5 \pi / 6$, and if $\varphi=2 \pi / 3$ then $|\alpha|=|\beta|$; if $\varphi=3 \pi / 4$ then either $|\alpha|=\sqrt{2}|\beta|$ or $|\beta|=\sqrt{2}|\alpha|$; finally, if $\varphi=5 \pi / 6$ then either $|\alpha|=\sqrt{3}|\beta|$, or $|\beta|=\sqrt{3}|\alpha|$ (cf. Corollary 2 of Theorem 1).

Now let us consider the case when there is a vector $\beta^{\prime} \in \mathfrak{M}$ having the same direction as $\beta$, with $\left|\beta^{\prime}\right|<|\beta|$. As we have just shown, for some integer $l \geq 0$ we must have

$$
l\langle\alpha, \alpha\rangle+2\left\langle\alpha, \beta^{\prime}\right\rangle=0 .
$$

Let $\varphi=2 \pi / 3$. Then from (12) we obtain $\left|\beta^{\prime}\right|=l|\alpha|$. This is a contradiction, for in this case $|\alpha|=|\beta|>\left|\beta^{\prime}\right|$ and $l \geq 1$. Thus, if $\varphi=2 \pi / 3$ there is no such $\beta^{\prime}$.

Assume now that $\varphi=3 \pi / 4$. Then from (12) we obtain $\left|\beta^{\prime}\right|=l|\alpha| / \sqrt{2}$. If $|\alpha|=\sqrt{2}|\beta|$ then $\left|\beta^{\prime}\right|=l|\beta|$. Since $l \geq 1$, this contradicts the initial assumption $\left|\beta^{\prime}\right|<|\beta|$. If $|\beta|=\sqrt{2}|\alpha|$ then $\left|\beta^{\prime}\right|=l|\beta| / 2$. Since $\left|\beta^{\prime}\right|<|\beta|, l=1$. Therefore, when $\varphi=3 \pi / 4$ we have $\beta^{\prime}=\beta / 2$.

Let us consider the remaining case $\varphi=5 \pi / 6$. Equality (12) yields $\left|\beta^{\prime}\right|=l|\alpha| / \sqrt{3}$. If $|\alpha|=\sqrt{3}|\beta|$ then $\left|\beta^{\prime}\right|=l|\beta|$. This is impossible, since $l \geq 1$. Assume now that $|\beta|=\sqrt{|\alpha|}$. Then $\left|\beta^{\prime}\right|=l|\beta| / 3$. Since $\left|\beta^{\prime}\right|<|\beta|$, either $l=1$ or $l=2$. Thus, if $\varphi=5 \pi / 6$ then $\beta^{\prime}$ may be either $\beta / 3$ or $2 \beta / 3$. Theorem 1 is completely proved.

\section{§3. Perturbation theory}

Together with the Hamiltonian system (3) let us consider the following system containing a parameter $\varepsilon$ :

$$
\begin{gathered}
\dot{x}_{s}=\partial H / \partial y_{s}, \quad \dot{y}_{s}=-\partial H / \partial x_{s}, \quad s=1, \ldots, n \\
H=H_{0}+\varepsilon H_{1}, \quad H_{0}=T, \quad H_{1}=V .
\end{gathered}
$$


As shown in [18], one can carry over to these systems the classical scheme of perturbation theory and relate the results of this theory with the problem on the existence of a complete set of independent integrals (cf. [19], Chapters V and IX). The results in this section were announced in [18].

Following the idea of the classical perturbation theory, let us attempt to find a formal canonical transformation $x, y \mapsto u, v$, analytic with respect to $\varepsilon$, of the form

$$
y=\partial S / \partial x, \quad u=\partial S / \partial v, \quad S=S_{0}(v, x)+\varepsilon_{1} S_{1}(v, x)+\cdots,
$$

carrying the Hamiltonian $H_{0}+\varepsilon H_{1}$ into a function $K_{0}(v)+\varepsilon K_{1}(v)+\cdots$ independent of the new $u$-coordinates. Let us set $S_{0}=(v, x)$; then for $\varepsilon=0$ we shall have the identity map. The generating function $S$ must satisfy the Hamilton partial differential equation

$$
H_{0}(\partial S / \partial x)+\varepsilon H_{1}(x)=K_{0}(v)+\varepsilon K_{1}(v)+\cdots .
$$

Hence we obtain an infinite chain of equations for the successive determination of $S_{1}, S_{2}, \ldots$ and $K_{1}, K_{2}, \ldots$ :

$$
\begin{gathered}
\langle v, \partial S / \partial x\rangle+H_{1}(x)=K_{1}(v), \\
\langle v \cdots \cdots \cdots \cdots \cdots \cdots \cdots \cdots \cdots \cdots \cdots \cdots \\
\left\langle v, \frac{\partial \dot{S}_{m}}{\partial x}\right\rangle+\frac{1}{2} \sum_{p+q=m}\left\langle\frac{\partial \dot{S}_{p}}{\partial x}, \frac{\partial \dot{S}_{q}}{\partial x}\right\rangle=K_{m}(v),
\end{gathered}
$$

Let us study the solvability of the first equation of this system. We write down formula (1) for the potential $H_{1}$ in a more convenient form for subsequent computations:

$$
H_{1}=\sum h^{a} \exp (a, x) \text {. }
$$

Here the summation is taken over a finite set of vectors $a \in \mathfrak{M}$. We assume that $a \neq 0$; the constant term in this formula may be associated with the function $K_{1}$. We shall seek a solution as a sum of exponents:

$$
S_{1}=\sum S_{1}^{a} \exp (a, x) .
$$

Then, clearly,

$$
S_{1}^{a}=-h^{a} /\langle v, a\rangle .
$$

The coefficients of $S_{1}$ are not defined on the "resonant" hyperplanes $\langle v, a\rangle=0$, $a \in \mathfrak{M}$. We denote the union of all those hyperplanes by $\boldsymbol{B}_{1}$, and call it the first-order secular set.

The equation for $S_{2}$ has the same form as $S_{1}$. The function

$$
\frac{1}{2}\left\langle\frac{\partial S_{1}}{\partial x}, \frac{\partial S_{1}}{\partial x}\right\rangle
$$

is a finite sum of exponents; however, its coefficients already depend on the new impulses $v$. We shall seek $S_{2}$ as the sum

$$
\sum S_{2}^{\tau} \exp (\tau, x)
$$

We put into $K_{2}$ the terms of (16) independent of $x$. From the second equation of (14) we find, taking (15) into account, that

$$
S_{2}^{\tau}=-\frac{1}{2\langle v, \tau\rangle} \sum_{\sigma+\delta=\tau} \frac{\langle\sigma, \delta\rangle h^{\sigma} h^{\delta}}{\langle v, \sigma\rangle\langle v, \delta\rangle} .
$$


The second-order secular set $\boldsymbol{B}_{2}$ is defined as the set of all $v \in \mathbf{R}^{n}$ such that $\langle v, \tau\rangle=0$ $(\tau \neq 0)$ and $\langle v, \tau\rangle S_{2}^{\tau} \not \equiv 0$.

The equations for $S_{3}, S_{4}, \ldots$ are solved successively by the same scheme. Let us set

$$
S_{m}=\sum=S_{m}^{\tau}(v) \exp (\tau, x), \quad S_{m}^{\tau} \not \equiv 0 .
$$

The coefficients in this sum are found by the following recurrent formula:

$$
S_{m}^{\tau}=-\frac{1}{2\langle v, \tau\rangle} \sum_{\substack{p+q=m \\ \sigma+\delta=\tau}}\langle\sigma, \delta\rangle S_{p}^{\sigma} S_{q}^{\delta},
$$

which follows form (14) and (17).

Let us introduce the $m$ th order secular set $\boldsymbol{B}_{m}$. It consists of all the $v \in \mathbf{R}^{n}$ such that

(1) $\langle v, \tau\rangle=0, \tau \neq 0$, and

(2) $\langle v, \tau\rangle S_{m}^{\tau}(v) \neq 0$.

Let us set $\boldsymbol{B}=\bigcup_{1}^{\infty} \boldsymbol{B}_{k}$, and call it the secular set of the Hamiltonian system (13). The secular set is defined in $\mathbf{R}^{n}=\{v\}$. Identifying the Cartesian spaces $\mathbf{R}^{n}=\{v\}$ and $\mathbf{R}^{n}=\{y\}$, we obtain a set of points in the $\{y\}$-space. In the sequel we shall denote this set also by $\boldsymbol{B}$.

The convenience of introducing and studying the secular set is clear from the following assertion.

Proposition 4. Assume that the Hamiltonian system (3) has $n$ polynomial integrals with respect to the impulse with coefficients of the form (6). Then their leading homogeneous forms do not depend on the $x$-coordinate and are dependent functions at all the points of the set $\boldsymbol{B}$.

The structure of the secular set is described by

LEMMA 4 (MAIN LEMMA). Let all the assumptions of Theorem 3 hold. Then the secular set $\boldsymbol{B}_{k}$ contains the hyperplane $\langle k \alpha+\beta, y\rangle=0$. In particular, the secular set $\boldsymbol{B}$ consists of infinitely many distinct hyperplanes, and its closure contains the hyperplane $\langle y, \alpha\rangle=0$.

Since the leading homogeneous forms of the polynomial integrals are analytic functions in $\mathbf{R}^{n}=\{y\}$ (Proposition 4), Proposition 4 and the main lemma imply Theorem 3. Indeed, the Jacobian of the leading homogeneous forms is an analytic function in $\mathbf{R}^{n}=\{y\}$ vanishing on an infinite set of hyperplanes passing through the origin. Therefore the Jacobian is identically zero, and hence the leading homogeneous forms of the $n$ polynomial integrals are everywhere dependent. The main lemma will be proved in $\S 4$, and now we shall prove Proposition 4.

Let us make the following change in the Hamiltonian system (3):

$$
x \mapsto x, \quad y \mapsto y / \sqrt{\varepsilon}, \quad t \mapsto \sqrt{\varepsilon} t .
$$

After this substitution (3) becomes system (13) and the polynomial integral becomes $F+\sqrt{\varepsilon} \Phi$ (up to an unessential constant factor), where $F$ and $\Phi$ are analytic functions of $\varepsilon$. Clearly, $F$ and $\Phi$ are integrals of (13), and one of the independent terms $F_{0}=\left.F\right|_{\varepsilon=0}$ or $\Phi_{0}=\left.\Phi\right|_{\varepsilon=0}$ coincides with the leading homogeneous form of the original polynomial integral.

We shall prove a stronger version of Proposition 4 for a Hamiltonian system (13) having $n$ formally analytic integrals with respect to $\varepsilon$

$$
F^{(1)}=F_{0}^{(1)}+\varepsilon F_{1}^{(1)}+\cdots, \ldots, F^{(n)}=F_{0}^{(n)}+\varepsilon F_{1}^{(n)}+\cdots .
$$


The coefficients of these power series-the functions $F_{i}^{(j)}$-are finite sums of exponents

$$
\sum f^{b}(y) \exp (b, x)
$$

and the functions $f^{b}$ are analytic in $\mathbf{R}^{n}=\{y\}$. In particular, this is the form of the integrals of the Hamiltonian equations (13) obtained from polynomial integrals of (3) by the substitution (19). In the sequel we shall frequently use the following evident fact: two functions of the form (20) coincide for all the values of $x$ and $y$ if and only if all the coefficients $f^{b}$ coincide.

Let us show that the functions $F_{0}^{(1)}, \ldots, F_{0}^{(n)}$ do not depend on $x$. Indeed, let $\sum_{0}^{\infty} F_{s} e^{s}$ be a formal integral of (13). Clearly, $F_{0}$ is an integral of the "unperturbed" system with Hamiltonian $H_{0}$. Assume that $F_{0}$ has the form of a finite sum (20). Differentiating $F_{0}$ along the solutions of the unperturbed problem, we obtain

$$
\sum f^{b}(y)\langle b, y\rangle \exp (b, x) \equiv 0 .
$$

Since (by the assumption of Theorem 3 ) the quadratic form $H_{0}$ is nondegenerate, we have

$$
\langle b, y\rangle=\left(b, \partial H_{0} / \partial y\right) \not \equiv 0
$$

for $b \neq 0$. Therefore $f^{b} \equiv 0$ for nonzero vectors $b$. Consequently, $F_{0}=f^{0}(y)$, as required.

Let us denote by $\{$,$\} the standard Poisson brackets given by the canonical$ coordinates $x_{1}, \ldots, x_{n} y_{1}, \ldots y_{n}$.

LeMma 5. Let $F=F_{0}+\varepsilon F_{1}+\cdots$ and $G=G_{0}+\varepsilon G_{1}+\cdots$ be two formal integrals of the equation (13) with coefficients of the form (20). Then the formal series

$$
\{F, G\}=\sum_{s}\left(\sum_{k+m=s}\left\{F_{k}, G_{m}\right\}\right) \varepsilon^{s}
$$

is equal to zero.

COROLlary. The polynomial integrals of the Hamiltonian system (3) are in involution.

We have already proved that the functions $F_{0}$ and $G_{0}$ do not depend on $x$. Consequently, $\left\{F_{0}, G_{0}\right\}=0$ and therefore the independent term of the formal series (21) equals zero. Let us make a canonical change of variables $x, y \mapsto u, v$ of the form

$$
y=v+\varepsilon \frac{\partial S^{\prime}}{\partial x}, \quad u=x+\varepsilon \frac{\partial S^{\prime}}{\partial v}, \quad S^{\prime}=S_{1}+\cdots+\varepsilon^{m-1} S_{m},
$$

where $S_{1}, \ldots, S_{m}$ are solutions of the first $m$ equations of (14). In the new variables the Hamiltonian function becomes

$$
K_{0}(v)=\varepsilon K_{1}(v)+\cdots+\varepsilon^{m} K_{m}(v)+\varepsilon^{m+1} K_{m+1}(u, v)+\cdots,
$$

and $K_{0}(v) \equiv H_{0}(v)$ and all the functions $K_{s}, s \geq 1$, are finite sums of exponents with respect to the variables $u$ whose coefficients are analytic in $\mathbf{R}^{n} \backslash$ $\left(\boldsymbol{B}_{1} \cup \cdots \cup \boldsymbol{B}_{m}\right)$. Let $\sum F_{s}(x, y) \varepsilon^{s}$ be a formal integral of the Hamiltonian system (13) with coefficients of the form (20). In the new variables $u, v$ the integral will have the same form $\sum F_{s}^{\prime}(x, y) \varepsilon^{s}$, and the functions $F_{s}^{\prime}$ will also be sums of exponents 
with respect to the $u$-coordinates, analytic with respect to $v$ in the domain $\mathbf{R}^{n} \backslash$ $\left(\boldsymbol{B}_{1} \cup \cdots \cup \boldsymbol{B}_{m}\right)$. Since for $\varepsilon=0$ the change (22) is the identity, $F_{0}^{\prime}$ will not depend on $u$ (by the previous arguments). The series $\sum F_{s}^{\prime} \varepsilon^{s}$ is a formal integral of the Hamiltonian system with Hamiltonian function $\sum K_{s} \varepsilon^{s}$. Consequently,

$$
\left\{\sum F_{s}^{\prime} \varepsilon^{s}, \sum K_{s} \varepsilon^{s}\right\}=\sum_{s}\left(\sum_{m+l=s}\left\{F_{m}^{\prime}, K_{l}\right\}\right) \varepsilon^{s} \equiv 0 .
$$

Equating to 0 the first $m$ coefficients of this series, we obtain

$$
\left\{F_{0}^{\prime}, K_{1}\right\}+\left\{F_{1}^{\prime}, K_{0}\right\}=0, \ldots,\left\{F_{m}^{\prime}, K_{0}\right\}+\cdots+\left\{F_{0}^{\prime}, K_{m}\right\}=0 .
$$

Since the functions $K_{0}, \ldots, K_{m}$ depend only on $v$ and $F_{1}^{\prime}, \ldots, F_{m}^{\prime}$ are sums of exponents with respect to $u$, these equalities imply that $F_{1}^{\prime}, \ldots, F_{m}^{\prime}$ are also independent of $u$. If now $G=\sum G_{s} \varepsilon^{s}$ is another formal integral of (13), then in the new variables $u, v$ the first $m$ coefficients of $\sum G_{s}^{\prime} \varepsilon^{s}$ are also independent of $u$. But then, clearly, the first $m$ coefficients of the formal series (21) vanish. Lemma 5 is proved.

Now let us prove that the functions $F_{0}^{(1)}, \ldots, F_{0}^{(n)}$ are independent at the points of $\boldsymbol{B}$. Assume that the point $y^{0}$ belongs to $\boldsymbol{B}$, and that the Jacobian

$$
\frac{\partial\left(F_{0}^{(1)}, \ldots, F_{0}^{(n)}\right)}{\partial\left(y_{1}, \ldots, y_{n}\right)} \neq 0
$$

at $y^{0}$. Let us consider the system of partial differential equations

$$
F_{0}^{(s)}\left(\frac{\partial S}{\partial x}\right)+\varepsilon F_{1}^{(1)}\left(\frac{\partial S}{\partial x}, x\right)+\cdots=F_{0}^{(s)}(v)+\sum_{k \geq 1} f_{k}^{(s)}(v) \varepsilon^{k}, \quad 1 \leq s \leq n,
$$

where the $f_{k}^{(s)}$ are unknown analytic functions for the time being. For $\varepsilon=0$ the equations will be satisfied if we set $S=(v, x)$. Since by (23) at $v=y^{0}$ the Jacobian of the functions $F_{0}^{(1)}, \ldots, F_{0}^{(n)}$ is nonzero, it follows that for given $f_{k}^{(s)}, k \geq 1$, the equations

$$
F_{0}^{(s)}(y)+\varepsilon F_{1}^{(s)}(y, x)+\cdots=F_{0}^{(s)}(v)+\varepsilon f_{1}^{(s)}(v)+\cdots
$$

have a unique formal series solution

$$
y=v+\sum_{k \geq 1} g_{k}(v, x) \varepsilon^{k},
$$

where the coefficients $g_{k}$ are finite sums of real exponents in the $x$-coordinates, analytic with respect to $v$ in a small neighborhood of $y_{0}$. We claim that the differential form

$$
y d x=\sum_{i=1}^{n} y_{i} d x_{i}
$$

is exact, i.e., it is the differential with respect to $x$ of a formal series $\sum S_{m}(x, v) \varepsilon^{m}$. This series is clearly a solution of (24). In order to prove that (25) is exact we first observe that the functions

$$
G_{i}=y_{i}-v_{i}-\sum_{k \geq 1} g_{i k}(v, x) \varepsilon^{k}, \quad i=1, \ldots, n,
$$


are in involution for fixed values of $v$. This is a simple consequent of the fact that the integrals $\sum F_{k}^{(s)} \varepsilon^{k}$ are in involution, $1 \leq s \leq n$ (Lemma 5). We have, further more,

$$
\left\{G_{i}, G_{j}\right\}=\sum_{k \geq 1}\left(\frac{\partial g_{j k}}{\partial x_{i}}-\frac{\partial g_{i k}}{\partial x_{j}}\right) \varepsilon^{k} \equiv 0 .
$$

Hence the form $y d x$ is closed and therefore exact in $\mathbf{R}^{2 n}=\{x, v\}$.

By a suitable choice of the functions $f_{k}^{(s)}(v)$ we may achieve that in the expansions of $g_{k}(v, x)$ into sums of exponents there are no terms independent of $x$. Indeed, the equation for $g_{1}$ has the following forms:

$$
J(v) g_{1}=f_{1}-F_{1},
$$

where $J$ is the Jacobi matrix of the functions $F_{0}^{(1)}, \ldots, F_{n}^{(n)} ; f_{1}$ is the vector with components $f_{1}^{(1)}, \ldots, f^{(n)}$, and $F_{1}$ is the vector with components $F_{1}^{(1)}, \ldots, F_{1}^{(n)}$. Let us set $f_{1}$ equal to the term in $F_{1}$ independent of $x$. The equations for $g_{k}$, for $k \geq 1$, have the form (26), and the right-hand side contains the functions $g_{m}$ and $f_{m}(m<k)$, which are already known.

From the equality

$$
\sum_{k \geq 1} g_{k}(v, x) \varepsilon^{k}=\frac{\partial}{\partial x} \sum_{k \geq 1} S_{k}(v, x) \varepsilon^{k}
$$

we can find the functions $S_{k}(v, x)$ as a sum of exponents also having no terms independent of $x$. Let $S$ denote the formal series

$$
(v, x)+\sum_{k \geq 1} S_{k}(v, x) \varepsilon^{k}
$$

Let us expand

$$
H_{0}(\partial S / \partial x)+\varepsilon H_{1}(x)
$$

in series in powers of $\varepsilon$; let $K_{0}+\varepsilon K_{1}+\cdots$ be this series. We show that the functions $K_{0}, K_{1}, \ldots$ do not depend on $x$. To this end we make a formal canonical transformation $x, y \mapsto u, v$ with generating function $S$. In the new variables the Hamiltonian has the form

$$
H_{0}(y)+\left.\varepsilon H_{1}(x)\right|_{u, v}=K_{0}^{\prime}(u, v)+\varepsilon K_{1}^{\prime}(u, v)+\cdots
$$

The coordinates $v_{1}, \ldots, v_{n}$ are first integrals of the systems with formal Hamiltonian $\sum K_{s}^{\prime} \varepsilon^{s}$ :

$$
\left\{v_{i}, \sum_{s} K_{s}^{\prime} \varepsilon^{s}\right\}=\sum_{s}\left\{v_{i}, K_{s}^{\prime}\right\} \varepsilon^{s} \equiv 0 .
$$

Therefore, $\left\{v_{i}, K_{s}^{\prime}\right\} \equiv 0$ for every $i$ and $s$, and consequently the functions $K_{s}^{\prime}$ do not depend on $u_{1}, \ldots, u_{n}$. Hence the functions $K_{s}=\left.K_{s}^{\prime}\right|_{u, x}$ depend only on the variables $v_{1}, \ldots, v_{n}$.

Thus the functions $S_{m}, m \geq 1$, satisfy (14) and are finite sums of exponents with analytic coefficients in a neighborhood of $y^{0} \in \boldsymbol{B}$. However, this contradicts the definition of the secular set $\boldsymbol{B}$. Proposition 4 is proved.

\section{§4. Structure of the secular set}

In this section we prove the main lemma of $\S 3$. Let $\prec$ be the lexicorgraphic order relation figuring in Theorem 3. 
LEMMA 6. The functions $S_{r}^{\tau}$ are identically zero for all $\tau \succ r \alpha$.

The proof is by induction on $r$. For $r=1$ the lemma follows from (15) and the definition of $\alpha$ as the greatest element of $\mathfrak{M}$. Assume that the lemma is valid for every $r \leq m$. The function $S_{r+1}^{\tau}$ is computed by (18). Assume that $\tau \succ(r+1) \alpha$. We shall prove that in every term in the right-hand side of (18) there must be a factor $S_{w}^{\tau}$ for $\tau \succ w \alpha, w \leq r$, which equals zero by the inductive hypothesis. Indeed, if $\sigma \preceq p \alpha$ and $\delta \preceq q \alpha$ then $\sigma+\delta \preceq(p+q) \alpha=(r+1) \alpha \prec \tau$. But this contradicts the summation condition $\sigma+\delta=\tau$. The lemma is proved.

LEMMA 7.

$$
S_{m}^{m \alpha}=-\frac{\langle\alpha \cdot \alpha\rangle}{2 m\langle v, \alpha\rangle} \sum_{p+q=m} p q S_{p}^{p \alpha} S_{q}^{q_{\alpha}} .
$$

Proof. Let us deduce (27) from (18), setting $\tau=m \alpha$. We consider only nonzero terms on the right-hand side. By Lemma 6 we have $\sigma \preceq p \alpha, \delta \preceq q \alpha$, and $\sigma+\delta=$ $m \alpha=(p+q) \alpha$, whence $\sigma=p \alpha$ and $\delta=q \alpha$, are required.

LEMMA 8.

$$
S_{m}^{m \alpha}=R_{m}\left(-\frac{\langle\alpha, \alpha\rangle}{\langle v, \alpha\rangle}\right)^{m-1}\left(S_{1}^{\alpha}\right)^{m}
$$

where

$$
R_{1}=1, \quad R_{m}=\sum_{p+q=m} \frac{p q R_{p} R_{q}}{2 m} .
$$

The proof is by induction on $m$. For $m=1,(28)$ is a trivial identity. Assume that Lemma 8 is valid for $m \leq r$. Then

$$
\begin{aligned}
S_{r+1}^{(r+1)^{\prime \prime}} & =-\frac{\langle\alpha, \alpha\rangle \sum_{p+q=r+1} p q R_{p} R_{q}}{2(r+1)\langle v, \alpha\rangle}\left(-\frac{\langle\alpha, \alpha\rangle}{\langle v, \alpha\rangle}\right)^{p+q-2}\left(S_{1}^{\alpha}\right)^{p+q} \\
& =R_{r+1}\left(-\frac{\langle\alpha, \alpha\rangle}{\langle v, \alpha\rangle}\right)^{r}\left(S_{1}^{\alpha}\right)^{r+1}
\end{aligned}
$$

The lemma is proved.

LEMMA 9.

$$
S_{m+1}^{m^{\alpha+\beta}}=-\frac{1}{\langle v, m \alpha+\beta\rangle} \sum_{\substack{p+q=m \\ p>0, q \geq 0}}\langle p \alpha, q \alpha+\beta\rangle S_{p}^{p \alpha} S_{q+1}^{q \alpha+\beta} .
$$

PRoof. From (18) it is not hard to deduce (for instance, by induction) that if $S_{k}^{\tau} \neq$ 0 then $\tau=\tau_{1}+\cdots+\tau_{k}$ with $\tau_{j} \in \mathfrak{M}, j=1, \ldots, k$. Let us set $\tau=m \alpha+\beta$ in (18). Then for the nonzero terms in (18) we have $\sigma=\tau_{1}+\cdots+\tau_{p}$ and $\delta=\tau_{1}^{\prime}+\cdots+\tau_{q}^{\prime}$, where $\tau_{j}, \tau_{k}^{\prime} \in \mathfrak{M}, j=1, \ldots ; p, k=1, \ldots, q$. Moreover, $\sigma+\delta=m \alpha+\beta$. Thus, by condition 2) of Theorem 3, we obtain either $\sigma=p \alpha$ and $\delta=(q-1) \alpha+\beta$, or $\sigma=(p-1) \alpha+\beta$ and $\delta=q \alpha$. To finish the proof it remains to use the symmetry of $(18)$ with respect to $\sigma$ and $\delta$. The lemma is proved.

Let us transform (30):

$$
\langle v, m \alpha+\beta\rangle S_{m+1}^{m \alpha+\beta}=\sum_{\substack{p+q=m \\ p>0, q \geq 0}}-\frac{\langle\alpha, q \alpha+\beta\rangle}{\langle v, q \alpha+\beta\rangle} p S_{p}^{p \alpha}\langle v, q \alpha+\beta\rangle S_{q+1}^{q \alpha+\beta} .
$$


We introduce the following notation:

$$
x_{m}=m S_{m}^{m \alpha}, \quad y_{m+1}=\langle v, m \alpha+\beta\rangle S_{m+1}^{m^{n+\beta}}, \quad l_{q}=-\frac{\langle\alpha, q \alpha+\beta\rangle}{\langle v, q \alpha+\beta\rangle} .
$$

Then (31) may be written as

$$
y_{m+1}=\sum_{\substack{p+q=m \\ p>0, q \geq 0}} l_{q} x_{p} y_{q+1} .
$$

LEMMA 10.

$$
y_{m+1}=w_{m} x_{1}^{m} y_{1}
$$

where

$$
w_{0}=1, \quad w_{m}=\sum_{\substack{p+q=m \\ p>0, q \geq 0}} p R_{p} h^{p-1} w_{q} l_{q}, \quad h=-\frac{\langle\alpha, \alpha\rangle}{\langle v, \alpha\rangle} .
$$

The proof is by induction on $m$, using (28).

Let us set $p R_{p}=r_{p}$. From (29) we we obtain

$$
r_{1}=1, \quad r_{m}=\sum_{\substack{p+q=m \\ p>0, q>0}} \frac{r_{p} r_{q}}{2} .
$$

Using the new notation, we have

$$
w_{m}=\sum_{\substack{p+q=m \\ p>0, q \geq 0}} r_{p} h^{q-1} w_{q} l_{q} .
$$

LEMMA $11.1-(1-2 z)^{1 / 2}=\sum_{1}^{\infty} r_{n} z^{n}$.

Corollary. $r_{m}=(2 m-3) ! ! / m ! !$ for $m>1$.

Proof of Lemma 11. By (33) the power series $f(z)=\sum_{1}^{\infty} r_{n} z^{n}$ satisfies $f^{2}-$ $2 f+2 z=0$. Since $f(0)=0$, we have $f(z)=1-(1-2 z)^{1 / 2}$, as required.

Lemma 12. For $m \geq 1$

$$
w_{m}=\sum_{k} \sum_{0=j_{0}<j_{1}<\cdots<j_{k}<m} r_{j_{1}-j_{0}} r_{j_{2}-j_{1}} \cdots r_{m-j_{k}} h^{m-k-1} l_{j_{0}} l_{j_{1}} \cdots l_{j_{k}} .
$$

This is easily deduced from (34) by induction on $m$.

Let us study the secular set. Since the vectors $\alpha$ and $\beta$ are linearly independent by assumption, the hyperplanes $\langle v, \alpha\rangle=0$ and $\Gamma_{m}=\{v:\langle v, m \alpha+\beta\rangle=0\}$ do not coincide. By Lemma 6, the functions $S_{r}^{0}$ are analytic almost everywhere on $\Gamma_{m}$ for $r<m+1$. In order to find out whether the hyperplane $\Gamma_{m}$ belongs to the secular set $\boldsymbol{B}_{m+1}$ we need to study the inequality $y_{m+1} \neq 0$. We use (32). In it we have $x_{1}=S_{1}^{\alpha}$ and $y_{1}=\langle v, \beta\rangle S_{1}^{\beta}$. The coefficients $S_{1}^{\alpha}$ and $S_{1}^{\beta}$ are nonzero by (15) and the definition of the vectors $\alpha$ and $\beta$. If $\langle v, \beta\rangle \equiv 0$ on $\Gamma_{m}$, then $\alpha$ and $\beta$ must be collinear. However, this is not so. Hence, $x_{1} \neq 0$ and $y_{1} \neq 0$. Therefore, $y_{m+1} \neq 0$ if and only if $w_{m} \neq 0$.

We consider two cases: $\langle\alpha, \alpha\rangle=0$ and $\langle\alpha, \alpha\rangle \neq 0$. In the first case we have $h=0$ and (by Lemma 8) $w_{m}=l_{0} l_{1} \cdots l_{m}$. Since (by the assumption of Theorem 3) $\langle\alpha, \beta\rangle \neq 0$; it follows that all the $l_{s}$ are nonzero and, therefore, $w_{m} \neq 0$. In the 
second case we introduce the number $\lambda=\langle\alpha, \beta\rangle /\langle\alpha, \alpha\rangle$. At the points of $\Gamma_{m}$ we have $\langle v, \beta\rangle=-m\langle v, \alpha\rangle$, and therefore $l_{q}=(\lambda+q) h /(q-m)$. Since in our case $h \neq 0,(35)$ implies that $w_{m}=0$ if and only if $\lambda$ is a root of the polynomial

$$
P_{m}(x)=\sum_{k} \sum_{0=j_{0}<j_{1}<\cdots j_{k}<m} r_{j_{1}}-j_{0} \cdots r_{m-j_{k}} \frac{\left(x+j_{0}\right) \cdots\left(x+j_{k}\right)}{\left(j_{0}-m\right) \cdots\left(j_{k}-m\right)}
$$

LEMMA 13.

$$
P_{m}(x)=\frac{(-1)^{m}}{m !} x\left(x+\frac{1}{2}\right) \cdots\left(\dot{x}+\frac{m-1}{2}\right) .
$$

In order to prove Lemma 13 we consider the new polynomials

$$
Q_{n}(y)=\left.\frac{P_{n}(x)}{-x}\right|_{x=n-y}, \quad Q_{0}=-\frac{1}{y} .
$$

LEMMA 14. The following recurrent relation holds:

$$
m Q_{m}=\sum_{\substack{p+q=m \\ p>0, q \geq 0}} r_{p}(q-y) Q_{q}
$$

Proof. Let us make the substitution $m-j_{l}=i_{k-l+1}$ in (36). Then

$$
\begin{aligned}
P_{m}(x)= & -\frac{x}{m} r_{m} \\
& +\sum_{k} \sum_{0<i_{1}<\cdots<i_{k}<m} r_{m-i_{k}} r_{i_{k}-i_{k-1}} \cdot r_{i_{1}} \frac{x}{-m} \cdot \frac{x+m-i_{k}}{-i_{k}} \ldots \frac{x+m-i_{1}}{-i_{1}} .
\end{aligned}
$$

Singling out the summation over $i_{k}$, we obtain

$$
P_{m}(x)=-\frac{x}{m} \sum_{i_{k}=0}^{m-1} P_{i_{k}}(x) r_{m-i_{k}}, \quad P_{0}=1 \text {. }
$$

This can be rewritten as

$$
m \frac{P_{m}}{x}=\sum_{k=0}^{m-1}(k-m-x) \frac{P_{k}}{x+m-k} r_{m-k} .
$$

Setting $x+m=y$ and $P_{n}=(n-y) Q_{n}$, we obtain

$$
m Q_{m}(y)=\sum_{k=0}^{m-1} r_{m-k}(k-y) Q_{k}(y), \quad Q_{0}=-\frac{1}{y}
$$

which is equivalent to (39).

LEMMA 15.

$$
\sum_{n=0}^{\infty} Q_{n} z^{n}=-\frac{1}{y}\left(\frac{1+(1-2 z)^{1 / 2}}{2}\right)^{2 y} .
$$

Proof. Let us set $g(z)=\sum_{0}^{\infty} Q_{n} z^{n}$. Relation (39) leads to the following differential equation for $g$ :

$$
z \frac{d g}{d z}=\left(z \frac{d g}{d z}-y g\right) f
$$


Here $f$ is the function in Lemma 11 . Solving this linear differential equation with initial condition $g(0)=-1 / y$, we obtain

$$
g(z)=-\frac{1}{y}\left(\frac{1+(1-2 z)^{1 / 2}}{2}\right)^{2 y},
$$

as required.

The function (40) is analytic for small $z$. Let us find its MacLaurin series. We set $g(z)=F\left(\varphi^{-1}(z)\right)$, where

$$
F(z)=-\frac{1}{y}\left(\frac{1+z}{2}\right)^{2 y}, \quad \varphi=\frac{1-z^{2}}{2} .
$$

Since $\varphi^{\prime}(1) \neq 0$, we may use Bürmann's theorem [20]:

$$
g(z)=g(0)+\left.\sum_{m=1}^{\infty} \frac{z^{m}}{m !} \cdot \frac{d^{m-1}}{d z^{m-1}}\right|_{z=1}\left[F^{\prime}(z)\{\Psi(z)\}^{m}\right],
$$

where

$$
\Psi(z)=\frac{z-1}{\varphi(z)}=-\frac{2}{1+z} .
$$

From (40) and (41) we easily obtain

$$
m ! Q_{m}=\left(\frac{2 m-1}{2}-y\right)\left(\frac{2 m-2}{2}-y\right) \cdots\left(\frac{m+1}{2}-y\right) .
$$

Going back to the old variable $x$ and using (38), we obtain (37) for the polynomial $P_{m}$. Lemma 13 is proved.

Let us proceed with the analysis of the secular set. Lemma 13 shows that $w_{m} \equiv 0$ on the hyperplane $\Gamma_{m}$ if and only if $\lambda=\langle\alpha, \beta\rangle /\langle\alpha, \alpha\rangle$ coincides with one of the following numbers: $0,-1 / 2, \ldots,-(m-1) / 2$. However, by the assumption of Theorem $3, \lambda \neq-m / 2$ for all integers $m \geq 0$. Consequently, the hyperplane $\Gamma_{m}=\{v:\langle v, m \alpha+\beta\rangle=0\}$ belongs to the secular $\boldsymbol{B}_{m+1} \subset \boldsymbol{B}$. As $m \rightarrow \infty$ the hyperplanes $\Gamma_{m}$ clearly accumulate towards the limit plane $\langle v, \alpha\rangle=0$. The proof of the main lemma is complete.

Received 15/JUNE/87

\section{BIBLIOGRAPHY}

1. Morikazu Toda, Nonlinear lattice dynamics, Iwanami Shoten, Tokyo, 1978; English transl., Theory of nonlinear lattices, Springer-Verlag, 1981.

2. O. I. Bogoyavlenskii, Methods in the qualitative theory of dynamical systems in astrophysics and gas dynamics, "Nauka", Moscow, 1980; English transl., Springer-Verlag, 1985.

3. M. Hénon, Integrals of the Toda lattice, Phys. Rev. B(3) 9 (1974), 1921-1923.

4. H. Flaschka, The Toda lattice. I, Phys. Rev. B(3) 9 (1974), 1924-1925.

5. S. V. Manakov, Complete integrability and stochastization of discrete dynamical systems, Zh. Ėksper. Teoret. Fiz. 67 (1974), 543-555; English transl. in Soviet Phys. JETP 40 (1974).

6. O. I. Bogoyavlensky [Bogoyavlenskii], On perturbations of the periodic Toda lattice, Comm. Math. Phys. 51 (1976), 201-209.

7. M. A. Olshanetsky [Ol'shanetskii] and A. M. Perelomov, Explicit solutions of classical generalized Toda models, Invent. Math. 54 (1979), 261-269.

8. Bertram Kostant, The solution to a generalized Toda lattice and representation theory, Advances in Math. 34 (1979), 195-338.

9. E. K. Sklyanin, Boundary conditions for integrable quantum systems, J. Phys. A 21 (1988), 23752389 . 
10. M. Adler and P. van Moerbeke, Kowalewski's asymptotic method, Kac-Moody lie algebras and regularization, Comm. Math. Phys. 83 (1982), 83-106.

11. The motion of a rigid body about a fixed point. (Collection dedicated to the memory of S. V. Kovalevskaya), Izdat. Akad. Nauk SSSR, Moscow, 1940.

12. George D. Birkhoff, Dynamical systems, Amer. Math. Soc., Providence, R. I., 1927.

13. Jean-Pierre Serre, a) Lie groups and lie algebras, Benjamin, New York, 1965. b) Algébres de lie semi-simple complexes, Benjamin, New York, 1966.

14. N. Bourbaki, Groupes et algèbres de lie, Chaps. 4-6, Actualités Sci. Indust., no. 1337, Hermann, Paris, 1968.

15. Sigurdur Helgason, Differential geometry, lie groups and symmetric spaces, Academic Press, 1978.

16. M. Adler and P. van Moerbeke, Completely integrable systems, Euclidean lie algebras and curves, Advances in Math. 38 (1980), 267-317.

17. Marcel Berger, Géométrie. Vols. 1-5, CEDIC, Paris, and Nathan Information, Paris, 1977.

18. V. V. Kozlov, On the theory of perturbations of Hamiltonian systems with noncompact invariant surfaces, Vestnik Moskov. Univ. Ser. I Mat. Mekh. 1988, no. 2, 55-61; English transl. in Moscow Univ. Mech. Bull. 43 (1988).

19. H. Poincaré, Les méthodes nouvelles de la mécanique céleste. Vols. I, II, Gauthier-Villars, Paris, 1892, 1893; reprint, Dover, New York, 1957.

20. E. T. Whittaker and G. N. Watson, $A$ course of modern analysis, 4th ed., Cambridge Univ. Press, 1927. 